\title{
Osteoclasts Lose Innate Inflammatory Reactivity to Metal and Polymer Implant Debris Compared to Monocytes/Macrophages
}

\author{
Jessica Yadav $^{3}$, Lauryn Samelko ${ }^{1,2}$, Phil Gilvar ${ }^{4}$, Kyron McAllister ${ }^{1}$ and Nadim James Hallab ${ }^{*}, 1$ \\ ${ }^{I}$ Department of Orthopedic Surgery, Rush University Medical Center, Chicago, IL 60612, USA \\ ${ }^{2}$ Department of Immunology, Rush University Medical Center, Chicago, IL 60612, USA \\ ${ }^{3}$ Department of Bioengineering, University of Illinois in Chicago, Chicago, IL 60612, USA \\ ${ }^{4}$ Medical College, University of Illinois in Chicago, Chicago, IL 60612, USA
}

\begin{abstract}
Long-term aseptic failures of joint replacements are generally attributed to implant debris-induced inflammation and osteolysis. This response is largely mediated by immune and bone cells (monocytes/macrophages and osteoclasts, respectively), that in the presence of implant debris (e.g. metal particles and ions), release pro-inflammatory cytokines such as IL-1 $\beta$, TNF- $\alpha$, and IL-6. The relative degree to which implant debris can illicit inflammatory response(s) from osteoclasts vs monocytes/macrophages is unknown, i.e. are osteoclasts a viable target for antiinflammatory therapy for implant debris? We investigated relative monocyte versus osteoclast inflammatory responses in a side-by-side comparison using implant debris from the perspective of both danger signaling (IL-1 $\beta$ ) and pathogenic recognition (TNF- $\alpha$ ) reactivity (Challenge Agents: Cobalt-alloy, Titanium-alloy, and PMMA particles, 0.9-1.8um-dia ECD and Cobalt, and Nickel-ions 0.01-0.1mM, all with and without LPS priming). Human monocytes/macrophages reacted to implant debris with $>100$ fold greater production of cytokines compared to osteoclast-like cells. Particulate Coalloy challenge induced $>1000 \mathrm{pg} / \mathrm{ml}$ of IL- $1 \beta$ and TNF- $\alpha$, in monocytes and $<50 \mathrm{pg} / \mathrm{mL} \mathrm{IL-1} \beta$ and TNF- $\alpha$ in osteoclasts. Cobalt ions induced $>3000 \mathrm{pg} / \mathrm{mL}$ IL- $1 \beta$ and TNF- $\alpha$ in monocytes/macrophages and $<50 \mathrm{pg} / \mathrm{mL}$ IL- $1 \beta$ and TNF- $\alpha$ in osteoclasts. The paracrine effect of supernatants from debris-treated monocytes/macrophages was capable of inducing greater osteoclastogenesis (TRAP+, $\mathrm{p}<0.06)$ and inflammation than direct debris challenge on osteoclasts. Our results indicate that as monocytes/macrophages differentiate into osteoclasts, they largely lose their innate immune reactivity to implant debris and thus may not be as relevant a therapeutic target as monocytes/macrophages for mitigating debrisinduced inflammation.
\end{abstract}

Keywords: DAMP, innate, implant debris, immune, ions, metal, monocyte/macrophages, osteoclasts, PAMP, particles.

\section{INTRODUCTION}

While total joint replacement implants generally do well over the short term, after 10-15 years they often require revision surgery due to aseptic loosening [1]. This has long been established as caused by local innate immune responses (granuloma) driving osteolysis/resorption of local bone [2]. Inflammatory cells such as monocytes and macrophages activated by particulate debris secrete cytokines that can induce osteolysis by promoting the activity of boneresorbing cells (osteoclasts) [3-5]. Thus, activated macrophages and monocytes play a role in osteoclastogenesis and osteoclast activation [3]. However, the degree to which implant debris impact osteoclasts directly versus indirectly through inflammatory cells such as monocytes remains uncertain [5]. It is unclear to what degree osteolysis occurs due to wear debris effects on osteoclasts directly or whether macrophage induced inflammatory responses effectively dominate this process when evaluated

*Address correspondence to this author at the Department of Orthopedic Surgery, Rush University Medical Center, 1735 W Harrison MC107, Chicago, IL 60612, USA; Tel: 312-942-7079; Fax: 312-942-8828;

E-mail:nhallab@rush.edu on an equal dose basis. Pro-inflammatory cytokines that are typically released by sentinel innate immune cells such as monocytes/macrophages cells are TNF- $\alpha$, IL- $1 \beta$, and IL-6 [6]. TNF- $\alpha$ has been established as a fundamentally critical cytokine in osteoclastogenesis [7-9]. IL-1 $\beta$ has also been found to influence osteoclasts formation, and plays an important role in osteolysis as well $[10,11]$. Are these cytokines produced to the same degree in osteoclasts as they are in monocytes/macrophages? We hypothesized that monocytes/macrophages will produce a greater proinflammatory effect than will osteoclasts when exposed to implant debris, and that this response by monocytes/macrophages will play a greater role in osteoclast formation than implant debris alone. We tested this hypothesis by challenging monocytes/macrophages and osteoclasts with metal particles, polymeric bone cement particles, metal ions, danger signaling controls and pathogen molecular pattern controls and measured resulting differences in cytokine production. We also evaluated the effects of indirect paracrine monocyte/macrophage responses implant debris $v s$ direct exposure on osteoclastogenesis as measured by TRAP staining. We used TNF- $\alpha$ and IL- $1 \beta$ as signature cytokines of danger and pathogen innate immune responses, respectively, shown previously to have a direct 
effect on bone resorption $[4,9,11,12]$. Our objective was to determine if monocytes/macrophages and osteoclasts react similarly to implant debris via their cytokine release profile, and if implant-debris-activated monocytes/macrophages induce more osteoclastogenesis than implant debris challenged osteoclast precursors.

\section{MATERIALS AND METHODS}

\section{Cell Culture}

Blood samples were obtained intravenously from $n=8$ healthy volunteers ( 3 females, 5 males, average age 26, normal $\mathrm{BMI}<24$, with no implants or health conditions) after institutionally approved informed consent (Rush University IRB). Peripheral blood mononuclear cells (PBMCs) were isolated by Ficoll gradient separation and collected for further purification. Peripheral blood CD14+ monocytes were isolated from PBMCs by negative selection using a magnetic bead antibody cocktail to CD3, CD7, CD16, CD19, CD56, CD123 and Glycophorin A (Miltenyi Biotec). Isolated human primary monocytes were assessed for $>90 \%$ purity using FACS. Freshly isolated human primary monocytes/macrophages were treated with Co-alloy and Tialloy particles as described below. Osteoclasts were challenged with metal particles after 6-7 days of differentiation. Osteoclasts precursors were generated using PBMC derived monocytes incubated in media (RPMI-1640 with $10 \%$ human AB serum) with $100 \mathrm{ng} / \mathrm{ml}$ RANKL (R\&D Systems), and $50 \mathrm{ng} / \mathrm{ml}$ macrophage colony-stimulating factor (M-CSF) (R\&D Systems), for 6-7 days, at $37^{\circ} \mathrm{C}$ and $5 \% \mathrm{CO}_{2}$ in a 48 -well plate, at 300,000 cells/well, with new media every other day, and supernatant collected on day 8 .

Visually confirmed osteoclast-like differentiation images were taken during the differentiation process, where monocytes/macrophages at day 1 (immediately proceeding isolation and plating) were small, round, adherent, and nearly confluent (Fig. 1A). After a week of incubation with RPMI-1640, M-CSF, and RANK-L, and replacing this media every other day, the monocytes/macrophages fused to become large multi-nucleated osteoclasts (Fig. 1B). Osteoclast-like differentiated PBMCs were verified by the appearance of multinucleated cells, and also by TRAPpositive staining. Fig. (1D) shows that osteoclasts were TRAP positive by day 7 . The qualitative similarity of osteoclast-like cells exposed to Co particles was also observed (Fig. 1C).

To test the effect of supernatants from debris-activated monocytes/macrophages vs direct debris challenge on osteoclastogenesis, monocytes/macrophages from $n=3$ subjects were plated in a 96-well plate in media with $10 \%$ human $\mathrm{AB}$ serum and stimulated overnight with the challenge agents. Simultaneously, a second portion was plated separately in a 96 -well plate in media with $10 \%$ human AB serum, $100 \mathrm{ng} / \mathrm{ml}$ RANKL and $50 \mathrm{ng} / \mathrm{ml} \mathrm{M-CSF}$ (48 hours), to produce osteoclast-precursors/osteoclasts. The monocyte supernatants were collected after 24 hrs challenge, and used to challenge osteoclasts for 48 hours (compared to direct implant debris challenge). Osteoclasts were TRAP+ stained (Sigma-Aldrich) and analyzed on an ELISA plate reader (450 $\mathrm{nm}$, optical density, OD).

\section{Cells Challenge}

The cells were stimulated with the appropriate challenge agents adjusted to challenge the cells at a ratio of 10 particles per cell (e.g. 3 million particles per well: for $3 \times 10^{5}$ cells/well in 48 well plates (verified by hemacytometry): Co-alloy particles (ASTM F-75, Size=0.9 $\mu \mathrm{m}$ ECD, Dose 10:1=particles:cell or approximately $30 \mu \mathrm{g} / \mathrm{mL})$, Ti-alloy particles (Ti-6Al-4V, ASTM F-136, Size=1.2 $\mu \mathrm{m}$ ECD, 10:1=particles:cell or approximately $30 \mu \mathrm{g} / \mathrm{mL}$ ), Cobalt ions $\left(\mathrm{CoCl}_{2}\right.$, Dose 0.01-0.1 mM), Nickel ions $\left(\mathrm{NiCl}_{3}\right.$, Dose: 0.01 $0.1 \mathrm{mM}$ ), Polymethlymethacrylate Bone Cement (PMMA, made from PalacosTM Zimmer Inc, Size $=1.8 \mu \mathrm{m}$ ECD, Dose: 10:1=particles:cell or approximately $30 \mu \mathrm{g} / \mathrm{mL}$ ), and innate danger signal immune response positive controls, Alum $(350 \mu \mathrm{g} / \mathrm{mL}$, Sigma St Louis) and Nigericin $(10 \mu \mathrm{M}$, Sigma St Louis). All particles were $>97 \%$ less than $5 \mu \mathrm{m}$ in size and commercially obtained (Bioengineering Solutions Inc, Oak Park, IL). Combined pathogen associated molecular pattern, (PAMP) and dangler associated molecular patterns (DAMP) were used to co-challenge cells by "priming" cells with LPS (50 ng/ml) for 2-3 hours then adding DAMP associated debris challenge agents for the remaining 22 hours of challenge prior to supernatant collection.

\section{Cytokine Analysis}

The supernatants were analyzed via ELISA (R\&D Systems) or Luminex (Millipore) assay for IL-1 $\beta$ and TNF$\alpha$.

\section{Statistical Analysis}

Statistical differences were determined by nonparametric analysis, ANOVA and Mann-Whitney test $(p<0.05$ indicated significance or as indicated in text) using GraphPad Prism 5.

\section{RESULTS}

\section{Implant Debris Challenged Osteoclasts vs Monocytes/Macrophages}

To determine the cytokine profile of the inflammatory response of monocytes/macrophages and osteoclasts to implant debris, human cells were challenged with particles and ions for 24 hours. Generally, there was $>100$ fold greater production of IL-1 $\beta$ (Fig. 2A, B) and TNF- $\alpha$ (Fig. 3A, B) by monocytes/macrophages compared to osteoclasts. IL-1 $\beta$ and TNF- $\alpha$ values were dramatically and significantly higher for all conditions in monocytes/macrophages when compared to osteoclasts $(\mathrm{p}<0.05)$, especially for Co-alloy particles $(\mathrm{p}=$ $0.0139)$, Co ions $(\mathrm{p}=0.0057), \mathrm{Ni}$ ions $(\mathrm{p}=0.0195)$, and PMMA particles $(\mathrm{p}=0.0139)$. Metal ions induce higher cytokine secretion in monocytes/macrophages than particles for both IL- $1 \beta$ and TNF- $\alpha$, i.e. monocytes/macrophages secreted more IL- $1 \beta$ (approx 5000pg/mL) compared to TNF$\alpha(<3000 \mathrm{pg} / \mathrm{mL})(\mathrm{p}<0.05)$. Metal ions ( $v s$ particles $)$ were also the more potent immunogen for osteoclasts which secreted more TNF- $\alpha$ (approx 200pg/mL) than IL-1 $\beta$ $(<20 \mathrm{pg} / \mathrm{mL})$ (Figs. 2A, B, 3A, B). Osteoclast secretion of IL$1 \beta$ was below or at detection limits of the assay indicating loss of this inflammasome response [13]. Surprisingly, osteoclasts did produce detectable levels of TNF- $\alpha$, to challenge with Ni ions $(\mathrm{p}=0.04)$ and PMMA particles $(\mathrm{p}=$ 0.02). 

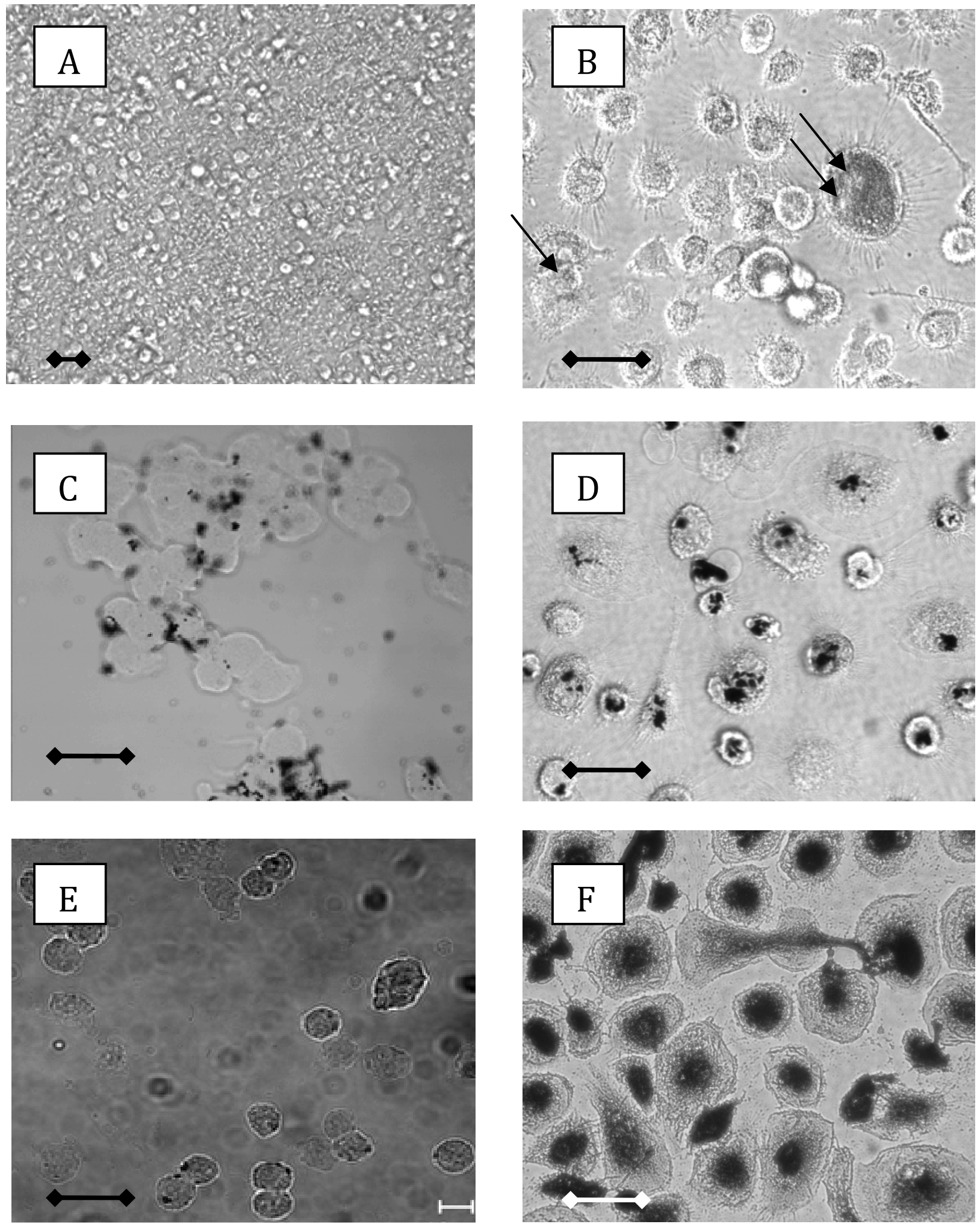

Fig. (1). Osteoclast maturation and particle challenge: Light microscopy was used to image monocytes/macrophages and osteoclasts. Peripheral blood mononuclear cells (PBMCs) are obtained from human whole blood, and monocytes/macrophages are isolated from the PBMCs and plated in 96-well plates. (A) Freshly isolated monocytes/macrophages at day 1, (B) Large osteoclast-like cells (some likely multinucleated, arrows) formed at day 6-7 after $100 \mathrm{ng} / \mathrm{ml}$ RANKL and 50ng/ml M-CSF treatment, (C) Monocytes/macrophage with Coalloy particles at 8 hours post-challenge, (D) Osteoclast-like cells 24 hours post challenge with Co-alloy particles showing phagocytosis, (E) Non-TRAP positive monocytes/macrophages, (F) TRAP-positive stained osteoclast-like cells (i.e. post RANKL/M-CSF stimulation), (Note: Bar = approx 50 microns, original mag A: 200x, B-F: 600x). 


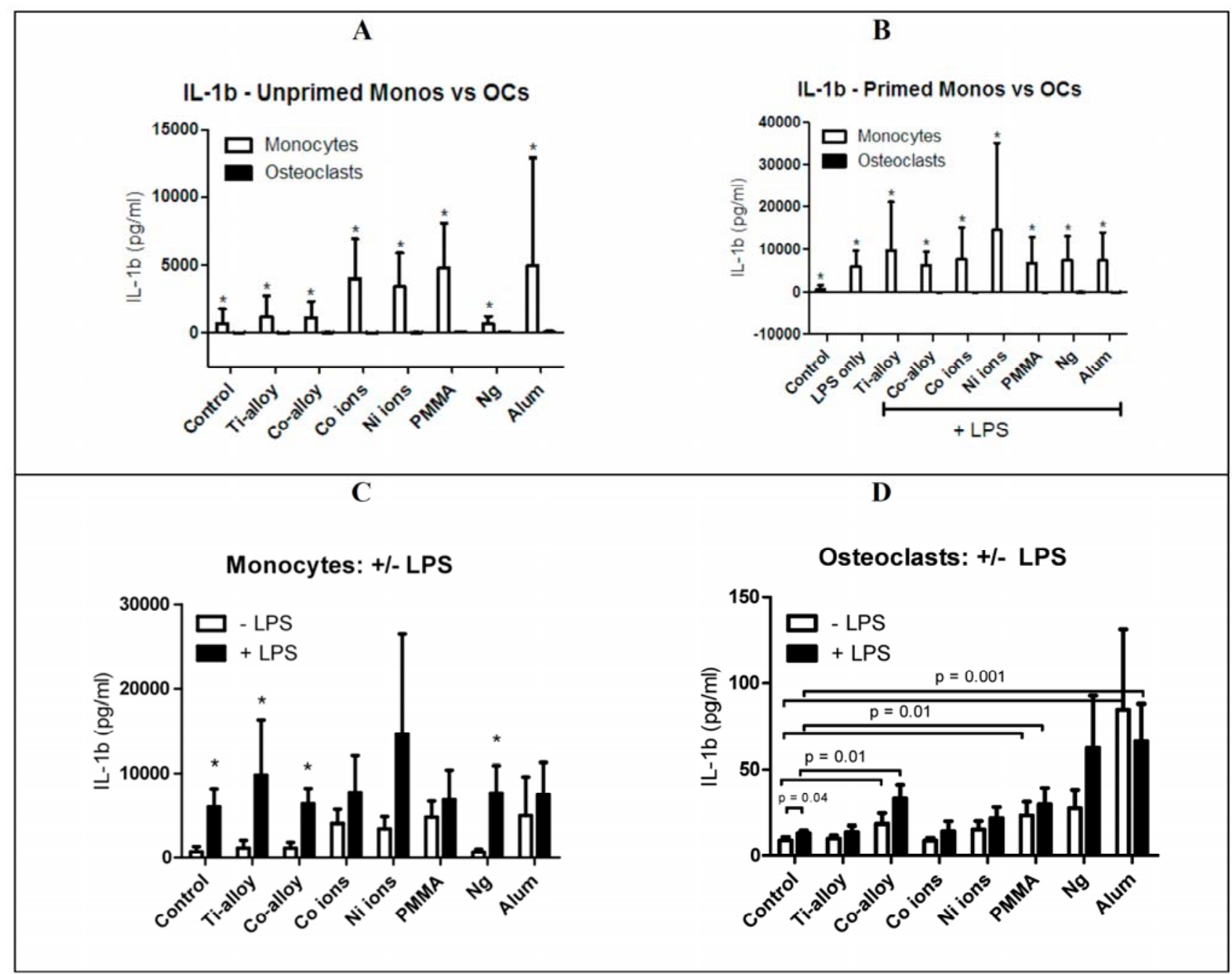

Fig. (2). IL-1 $\beta$ secretion from monocytes/macrophages (n=3-4) and osteoclasts $(n=8)$, challenged with particles and ions with or without LPS for 24 hours. PBMCs are obtained from human whole blood, monocytes/macrophages are isolated from PBMCs, and challenged with metal particles or ions, with and without LPS, for 24 hours. To form osteoclasts, monocytes/macrophages are cultured with M-CSF (50 ng/ml) and RANKL (100 ng/ml) for 6-7 days, and challenged with metal particles or ions, with and without LPS, for 24 hours. 24 hours later, IL-1 $\beta$ cytokine secretion is assessed in supernatants of each condition. (A) Monocytes secreted $>3$ orders of magnitude more IL-1 $\beta$ compared to osteoclasts, where osteoclast responses (black bars) are barely evident (asterisks indicate $\mathrm{p}<0.0002$ of monocytes vs osteoclasts). (B) Comparison between the cytokine secretion of primed/co-challenged monocytes/macrophages and osteoclasts (asteriks indicate statistical significance of monocyte $v s$ osteoclasts comparison for each challenge condition at $\mathrm{p}<0.0001$ ), (C) Comparison between the cytokine secretion of primed/co-challenged and unprimed (no LPS) monocytes/macrophages, and (D) Comparison between the cytokine secretion of primed/co-challanged and unprimed osteoclasts. Note: Statiscal comparison of adjacent bars (black vs white) are indicated by "** corresponding to $\mathrm{p}<0.05$ or as indicated.

\section{Implant Debris and LPS Challenged Osteoclasts and Monocytes/Macrophages}

Osteoclasts produced significantly less IL- $1 \beta$ and TNF- $\alpha$ compared to monocytes/macrophages in response to either LPS primed or unprimed debris challenge. Generally, monocytes/macrophages challenged simultaneously with LPS and implant debris secreted higher amounts of IL-1 $\beta$ and TNF- $\alpha$ compared to challenge with implant debris alone (Figs. 2C, D, 3C, D). However these increases were generally not significantly greater than LPS alone, demonstrating the powerful if not overwhelming effect of LPS on debris-related immune reactivity at the levels of LPS used in this study. Statistically significant increase in IL-1 $\beta$ and TNF- $\alpha$ from unprimed to LPS primed monocytes/macrophages challenged with Ti-alloy and Coalloy particles $(\mathrm{p}=0.02)$ was observed. In contrast, there was a non-significant increase between +LPS primed and non-primed monocytes/macrophages treated with $\mathrm{Co}$ or $\mathrm{Ni}$ ions or PMMA particles, suggesting metal ions are a more potent form of immunogen than particles (i.e. a maximal response was likely induced by ions alone, at the concentrations of challenges used). LPS primed osteoclasts challenged with particles and ions produced significantly more TNF- $\alpha$ than IL-1 $\beta$ indicating that TNF- $\alpha$ PAMPrelated responses may be retained by osteoclasts. Generally there were non-significant increases in IL-1 $\beta$ induced by particle and ion challenge in unprimed osteoclasts. Additionally, LPS primed osteoclasts plus particles and/or ions did not demonstrate significantly increases in IL- $1 \beta$ or 


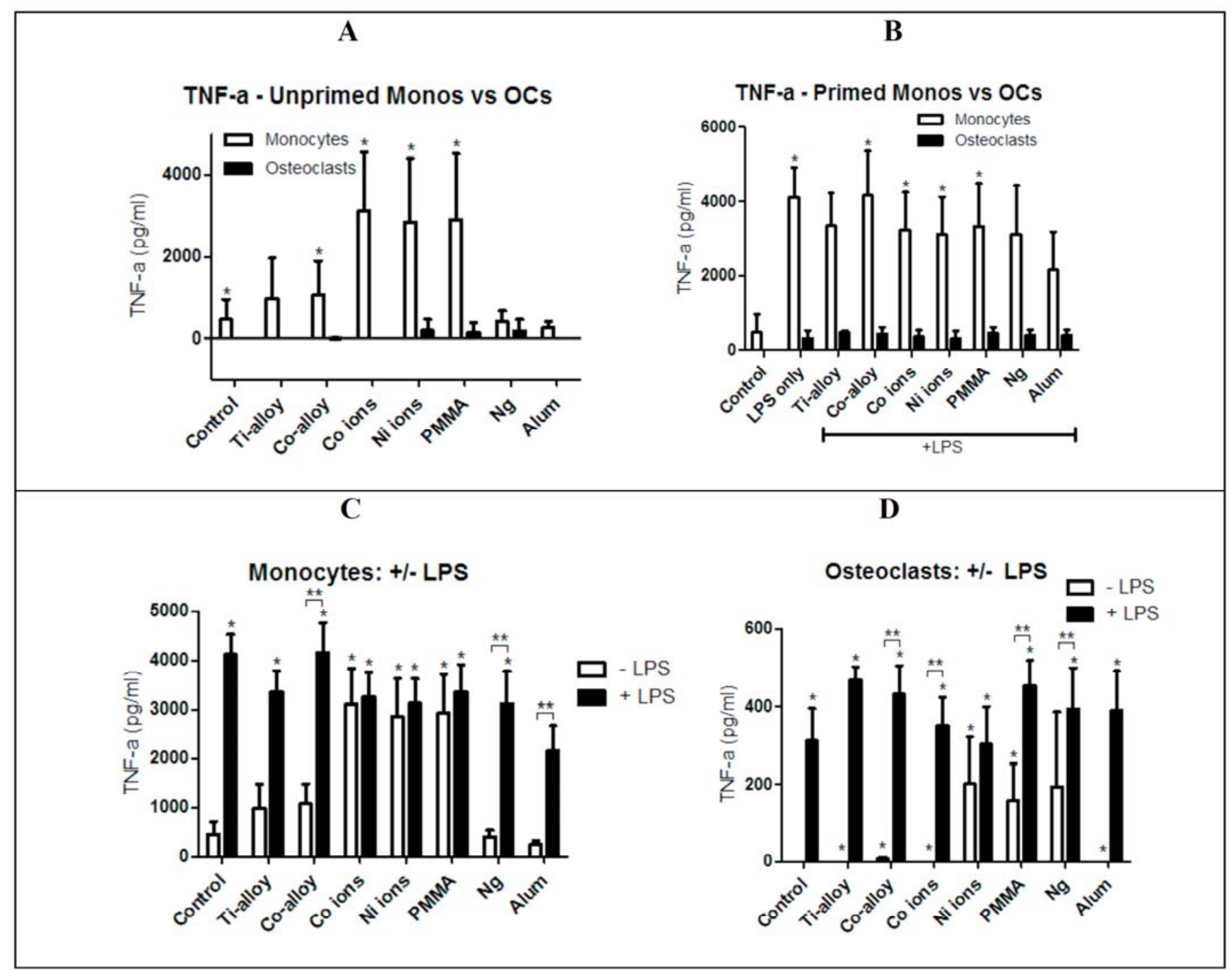

Fig. (3). TNF- $\alpha$ secretion from monocytes/macrophages $(n=3-4)$ and osteoclasts $(n=8)$, challenged with particles and ions with or without LPS for 24 hours. PBMCs are obtained from human whole blood, monocytes/macrophages are isolated from PBMCs, and challenged with metal particles or ions, with and without LPS, for 24 hours. To form osteoclasts, monocytes/macrophages are cultured with M-CSF (50 $\mathrm{ng} / \mathrm{ml})$ and RANKL (100 ng/ml) for 6-7 days, and challenged with metal particles or ions, with and without LPS, for 24 hours. 24 hours later, TNF- $\alpha$ cytokine secretion is assessed in supernatants of each condition. (A) Comparison between the cytokine secretion of unprimed (no LPS) monocytes/macrophages and osteoclasts (asteriks indicate statistical significance of monocyte $v s$ osteoclasts comparison for each challenge condition at $\mathrm{p}=0.0009)$, (B) Comparison between the cytokine secretion of primed/co-challenged monocytes/macrophages and osteoclasts (asteriks indicate statistical significance of monocyte $v s$ osteoclasts comparison for each challenge condition at $\mathrm{p}<0.0001)$, $(\mathbf{C})$ Comparison of TNF- $\alpha$ cytokine secretion from primed/co-challenged and unprimed monocytes/macrophages, and (D) Comparison of TNF- $\alpha$ cytokine secretion from primed/co-challenged and unprimed osteoclasts. Note: $\mathrm{Ti}=$ Titanium, Co $=$ Cobalt, PMMA = polymethylmethacrylate (bone cement), $\mathrm{Ng}=$ Nigericin, Alum=Alum vacine adjuvant; ${ }^{*}=\mathrm{p}<0.05$ comparing corresponding values between monocytes/macrophages and osteoclasts, $* *=p<0.05$ between the two highlighted conditions.

TNF- $\alpha$ over that of LPS challenge alone (except for Co-alloy and PMMA particle induced IL-1 $\beta$, see Figs. 2C, D).

\section{Implant Debris and Osteoclastogenesis-Like Responses}

We determined the amount of TRAP+ in PBMCs derived osteoclast-like primary human as a determinant of osteoclastogenesis. We observed greater TRAP staining in response to particle and ion challenge $(\mathrm{p}<0.05)$ as shown in Fig. (4) as positive percentage increases in TRAP staining over non-treated controls osteoclasts-like cells from the same individual. There was significant variability between the individual tests where, among the $n=3$ tested, there were cases of significant increases in TRAP+ to particle and/or metal ion challenge on an individual basis. On a group basis there were significant increases in the amount of TRAP staining when osteoclasts were challenged with Co $(p=0.1)$ and $\mathrm{Ni}(\mathrm{p}=0.02)$ ions and LPS $(\mathrm{p}=0.1)$, over controls.

\section{Monocyte/Macrophage Paracrine Induced Osteoclasto- genic-Like Activity}

Supernatants from debris-challenged monocytes/macrophages were used to challenge differentiated osteoclasts-like cells derived from the PBMCs of individual to compare these responses with implant debris induced effects on 


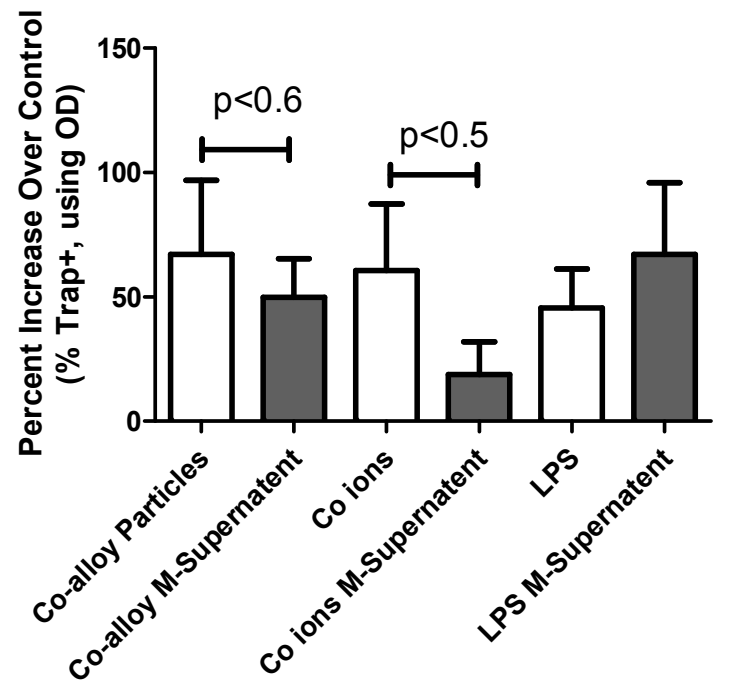

(A) Subject \#1

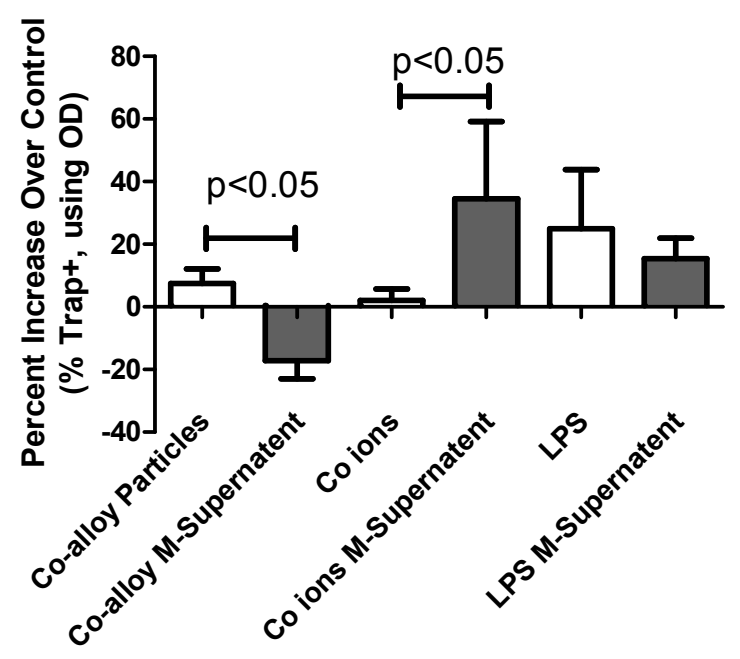

Subject \#3

(C)

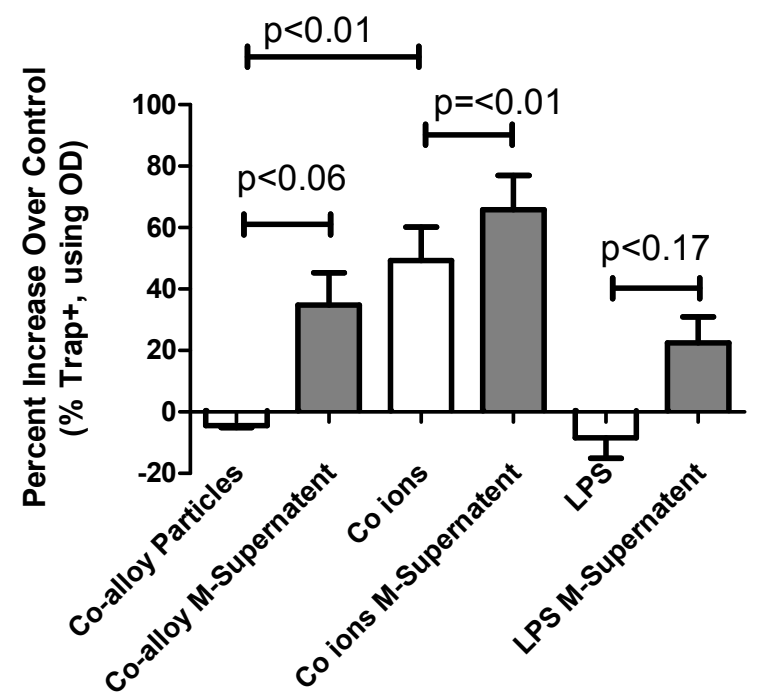

(B)

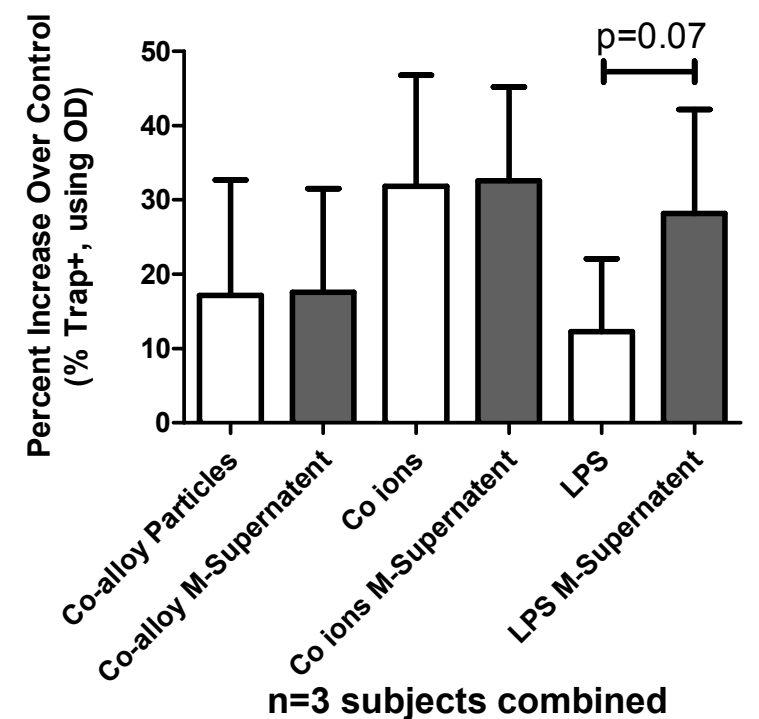

(D)

Fig. (4). Increases in TRAP staining of primary osteoclasts were stimulated with either metal implant debris directly or with the supernatants from implant-debris-activated-monocytes/macrophages. (A-C) The results indicated highly variable individual responses where only two individuals had significant increases in the amount of osteoclast TRAP staining when treated with metal ion supernatants of monocytes/macrophages-treated cell (from the same individual). (D) However when this behavior was averaged for $n=3$ individuals this increase was not apparent, given the variability in responses. And while there was on average 2-fold greater increase in TRAP positive staining for the osteoclasts treated with Co ions than (directly or with supernatants) vs Co-alloy particles, these increases were not significant (except for LPS, $\mathrm{p}=0.07$, t-test). Monocytes/macrophages were isolated from human PBMCs and divided in two portions. The osteoclasts were challenged for 24 hours, and average TRAP staining was measured via optical density (OD)/well. Note: M-Supernatant indicates Monocyte/macrophage-supernatant (treated for the prior 24 hours with either Co ions or particles or LPS).

osteoclastogenesis-like activity (TRAP+). There was individual variability between the different primary human PBMC cultures derived from $n=3$ individuals. In one of the three subjects tested, the supernatants from autologous monocytes/macrophages treated with Co-particles and Co ions resulted in significant increases in osteoclastogenesislike activity over debris challenge alone, (Fig. 4A). However due to the small sample size, on average $(n=3)$ the amount of TRAP+ staining between osteoclast-like cells treated with monocyte supernatants and those treated with implant debris directly did not rise to the level of statistical significance
(Fig. 4B). However on average particles and ions increased osteoclastogenesis-like activity by $>15 \%$ compared to nonchallenged controls. Co ions induced an increase of more than $15 \%$ activity over Co-alloy treated primary osteoclasts. ( $\mathrm{p}=0.1$, ANOVA).

\section{DISCUSSION}

We found that monocytes/macrophages produce well over an order of magnitude greater inflammatory responses (IL-1 $\beta$ and TNF- $\alpha$ ) compared to osteoclasts responses to 
metal- and polymer-particle and ion challenge, where metal ions challenge induced the greatest inflammatory responses in both monocytes/macrophages and osteoclast-like cells, after the positive control LPS. Even indirect debris-induced monocyte reactivity acting in a paracrine manner induced more osteoclastogenic-like activity (TRAP+) than direct debris challenge, in some individuals. Thus, the results of this study not only support our hypothesis that monocytes/macrophages will produce a greater inflammatory reaction to metal implant debris than will osteoclasts, but suggests danger signaling inflammasome-like (IL-1 $\beta$ ) reactivity is all but lost in osteoclast-like cells, whereas more pathogen related LPS (PAMP) recognition and responses $(\mathrm{TNF}-\alpha)$ are only dampened (relatively retained) in osteoclasts.

IL-1 $\beta$ and TNF- $\alpha$ are both well established proinflammatory cytokines that can contribute to bone resorption $[4,7,9,10,12,14]$. IL- $1 \beta$ is the signature cytokine of inflammasome-mediated DAMP responses, and TNF- $\alpha$ and IL-6 are generally proxies of NF-kB-mediated PAMP responses. Monocytes/macrophages demonstrated $>10$ fold greater IL- $1 \beta$ and TNF- $\alpha$ responses to implant debris challenge agents compared to osteoclasts, implying that as monocytes/macrophages differentiate into osteoclasts, they lose their ability to induce a strong danger signaling inflammatory response, probably due to role specialization. This is the first study that demonstrates the decreased ability of monocytes to respond to implant debris as they differentiate into osteoclasts. Osteoclasts were observed to secrete more TNF- $\alpha$ than IL- $1 \beta$ while monocytes/macrophages secreted more IL- $1 \beta$ than TNF- $\alpha$, consistent with past studies $[7,15]$ and suggest that osteoclasts preferentially lose Danger Signaling (inflammasome) responses compared to $\mathrm{NF}-\mathrm{\kappa B}$ pathway associated pathogen associated responses (i.e. PAMP receptor TLR4 recognition of LPS). A previous study by Stea et al. [16] demonstrated a positive correlation between wear debris and the cytokines IL-1 $\beta$, IL- 6 , and TNF- $\alpha$, and osteolysis. And our finding that TNF- $\alpha$ is more prominent in osteoclast cultures is in line with studies that state that TNF$\alpha$ is a fundamental cytokine in "mediating particle-driven osteoclastogenesis and osteolysis" [7]. These results also support work by Abu-Amer et al. who found that TNF- $\alpha$ is a primary osteoclastogenic cytokine in inflammatory osteoclastogenesis and that TNF- $\alpha$ recognition (by TNFr1) was required for optimal RANK-mediated osteoclastogenesis [14]. However, IL-1 $\beta$ has been shown to have almost the opposite effects on osteoclastogenesis, where early presence of IL- $1 \beta$ has been shown as inhibitory to osteoclast formation but synergizes with RANKL later on to promote differentiation [17]. This may indicate why more TNF- $\alpha$ and less IL- $1 \beta$ are necessary as osteoclastogenesis occurs.

Lipopolysaccharide (LPS, i.e. endotoxin) is a known pathogen associated molecular pattern (PAMP) associated with bone loss and osteoclast formation [8, 18, 19], and a known inducer of a strong inflammatory response in monocytes/macrophages through CD14 induced toll-like receptor 4 (TLR4) activation. The exact role LPS endotoxins play in the biological activity of implant debris is uncertain $[20,21]$. To test the potentiating effects of LPS on the proinflammatory response of monocytes/macrophages and osteoclasts, both were implant-debris-challenged when under unprimed conditions (LPS). In general, both monocytes/macrophages and osteoclasts challenged with implant debris under primed conditions secrete higher levels of IL-1 $\beta$ and TNF- $\alpha$, with monocytes/macrophages generally secreting more cytokine than osteoclasts. However, particles and ions alone also induced TNF- $\alpha$, supporting the results of Pearl et al. which demonstrate that TNF- $\alpha$ production occurs even in the absence of LPS [20]. This is also consistent with past studies that show endotoxins are not required for implant debris to elicit a danger signal (DAMP) mediated inflammatory responses, but LPS, when present, certainly potentiates and can dominate this response [18].

When comparing the direct effects of particles and ions on osteoclasts with the supernatants taken from particle/ion activated monocytes/macrophages we found evidence of particle/ion-directed and supernatant directed osteoclastogenesis in osteoclast precursor cells which is consistent with previous reports of particle/ion induced osteolysis and osteoclast formation [14, 15, 22-24]. Similarly to previous reports where LPS is shown to encourage differentiation of pre-osteoclast cell lines into bone-resorbing osteoclasts $[8,19]$, we found that on average LPS induced elevated TRAP+ osteogenic-like increases. Thus, LPS challenge induced an increased danger signal type inflammatory response in monocytes/macrophages but not osteoclasts (for particle and ion challenge). However, LPS challenge increased TNF- $\alpha$ (PAMP) in both debrischallenged monocytes/macrophages and osteoclasts. A noteworthy exception to this trend were $\mathrm{Co}$ and $\mathrm{Ni}$ ion challenge on monocytes/macrophages and osteoclasts (Nickel only) which may have already induced maximal PAMP-related inflammatory responses without the addition of LPS. Previous studies have demonstrated that TNF- $\alpha$ cultured with pre-osteoclasts can promote the amount of TRAP positive cells $[8,9,14,19]$. This is supported by our results which show that TNF- $\alpha$ is produced by debrisstimulated monocytes/macrophages, and that these supernatents of debris challenge cells can promote osteoclast formation in "pre-osteoclast-like" cells.

Limitations inherent to this study include those associated with the variability of primary human cells reactivity tested in vitro with simulated implant debris. Additionally, although there were low numbers of subjects studied $(n=3)$ to determine if reactivity to monocyte/debrissupernatants could surpass the osteoclastogenic effects of debris alone, there was evidence that this could occur and generally trends established that warrant further investigation and larger scale study.

\section{CONCLUSIONS}

In summary we found primary monocytes/macrophages exposed to metal and polymer particles and ion challenge have an order of magnitude greater inflammatory IL- $1 \beta$ and TNF- $\alpha$ responses (signature DAMP and PAMP cytokines, respectively) compared to autologous osteoclast-like cells. Additionally, the paracrine response by monocytes/macrophages exposed to implant debris can induce greater osteoclastogenic-like activity than direct osteoclast-debris exposure. The diminished osteoclast IL- $1 \beta$ response to implant debris relative to TNF- $\alpha$ suggests 
osteoclasts preferentially lose danger signaling (inflammasome) responses upon differentiation but retain TNF- $\alpha$ (pathogen recognition associated) responses which also play a role in bone homeostasis. Further study is warranted to determine how and why danger signaling responses in osteoclasts are lost. Generally the comparison of monocyte/macrophage $v s$ osteoclast innate immune responses to particulate and ionic implant debris indicates that monocytes/macrophages rather than osteoclasts dominate this immune response and may be a more relevant target to try to specifically inhibit this response in people with implant debris-induced inflammation.

\section{ABBREVATIONS}

$$
\begin{array}{ll}
\text { ASTM } & =\text { American Society of Testing and Materials } \\
\mathrm{Co} & =\text { Cobalt } \\
\mathrm{DAMP} & =\text { Danger associated molecular pattern } \\
\mathrm{ECD} & =\text { Equivalent spherical diameter } \\
\mathrm{IL}-1 \beta & =\text { Interleukin-1 beta } \\
\mathrm{IL}-6 & =\text { Interleukin-6 } \\
\text { LPS } & =\text { Lipopolysaccharide } \\
\mathrm{M}-\mathrm{CSF} & =\text { Macrophage colony stimulating factor } \\
\text { NF-KB } & =\text { Nuclear factor kappa-light-chain-enhancer of } \\
& \text { activated B cells } \\
\text { Ni } & =\text { Nickel } \\
\text { PAMP } & =\text { Pathogen-associated molecular pattern } \\
\text { PBMCs } & =\text { Peripheral blood mononuclear cells } \\
\text { PMMA } & =\text { polymethlymethacrylate (bone cement) } \\
\text { RANKL } & =\text { Receptor activator of nuclear factor kappa-B } \\
\text { TLR } & \text { ligand } \\
\text { TNF- } \alpha & =\text { Toll-like receptor } \\
\text { TRAP } & =\text { Tartrate-resistant acid phosphatase }
\end{array}
$$

\section{CONFLICT OF INTEREST}

The authors confirm that this article content has no conflict of interest.

\section{ACKNOWLEDGEMENTS}

We would like to acknowledge the support of the Crown Family Chair of Orthopedics and Dr Joshua Jacobs for his support and the National Institute of Arthritis and Musculoskeletal and Skin Diseases of the National Institutes of Health under Award Number R01AR060782-01A1. The content is solely the responsibility of the authors and does not necessarily represent the official views of the National Institutes of Health.

\section{REFERENCES}

[1] Kurtz S, Ong K, Lau E, Mowat F, Halpern M. Projections of primary and revision hip and knee arthroplasty in the United States from 2005 to 2030. J Bone Joint Surg Am 2007; 89(4): 780-5.
[2] Friedman RJ, Black J, Galante JO, Jacobs JJ, Skinner HB. Current concepts in orthopaedic biomaterials and implant fixation. Instr Course Lect 1994; 43: 233-55.

[3] Ingram JH, Kowalski R, Fisher J, Ingham E. The osteolytic response of macrophages to challenge with particles of Simplex P, Endurance, Palacos R, and Vertebroplastic bone cement particles in vitro. J Biomed Mater Res B Appl Biomater 2005; 75(1): 210-20.

[4] Wang JY, Wicklund BH, Gustilo RB, Tsukayama DT. Titanium, chromium and cobalt ions modulate the release of bone-associated cytokines by human monocytes/macrophages in vitro. Biomater 1996; 17(23): 2233-40.

[5] Tamaki Y, Sasaki K, Sasaki A, et al. Enhanced osteolytic potential of monocytes/macrophages derived from bone marrow after particle stimulation. J Biomed Mater Res B Appl Biomater 2008; 84(1): 191-204.

[6] Wang ML, Sharkey PF, Tuan RS. Particle bioreactivity and wearmediated osteolysis. J Arthroplasty 2004; 19(8): 1028-38.

[7] Clohisy JC, Hirayama T, Frazier E, Han SK, Abu-Amer Y. NF-kB signaling blockade abolishes implant particle-induced osteoclastogenesis. J Orthop Res 2004; 22(1): 13-20.

[8] Mormann M, Thederan M, Nackchbandi I, Giese T, Wagner C, Hansch GM. Lipopolysaccharides (LPS) induce the differentiation of human monocytes to osteoclasts in a tumour necrosis factor (TNF) alpha-dependent manner: a link between infection and pathological bone resorption. Mol Immunol 2008; 45(12): 3330-7.

[9] Wei S, Siegal GP. Mechanisms modulating inflammatory osteolysis: a review with insights into therapeutic targets. Pathol Res Pract 2008; 204(10): 695-706.

[10] Jimi E, Nakamura I, Duong LT, et al. Interleukin 1 induces multinucleation and bone-resorbing activity of osteoclasts in the absence of osteoblasts/stromal cells. Exp Cell Res 1999; 247(1): 84-93.

[11] Liu YP, Yu GR, Li K, Yuan F. Is there another possible approach to inhibit wear particles-induced inflammatory osteolysis? Med Hypotheses 201; 76(2): 280-2.

[12] Green TR, Fisher J, Matthews JB, Stone MH, Ingham E. Effect of size and dose on bone resorption activity of macrophages by in vitro clinically relevant ultra high molecular weight polyethylene particles. J Biomed Mater Res 2000; 53(5): 490-7.

[13] Caicedo MS, Desai R, McAllister K, Reddy A, Jacobs JJ, Hallab NJ. Soluble and particulate Co-Cr-Mo alloy implant metals activate the inflammasome danger signaling pathway in human macrophages: A novel mechanism for implant debris reactivity. J Orthop Res 2008; 27(7): 847-54.

[14] Abu-Amer Y, Abbas S, Hirayama T. TNF receptor type 1 regulates RANK ligand expression by stromal cells and modulates osteoclastogenesis. J Cell Biochem 2004; 93(5): 980-9.

[15] Clohisy JC, Frazier E, Hirayama T, Abu-Amer Y. RANKL is an essential cytokine mediator of polymethylmethacrylate particleinduced osteoclastogenesis. J Orthop Res 2003; 21(2): 202-12.

[16] Stea S, Visentin M, Granchi D, et al. Cytokines and osteolysis around total hip prostheses. Cytokine 2000; 12(10): 1575-9.

[17] Lee B, Kim TH, Jun JB, et al. Direct inhibition of human RANK+ osteoclast precursors identifies a homeostatic function of IL-1beta. J Immunol 2010; 185(10): 5926-34.

[18] Itoh K, Udagawa N, Kobayashi K, et al. Lipopolysaccharide promotes the survival of osteoclasts via Toll-like receptor 4, but cytokine production of osteoclasts in response to lipopolysaccharide is different from that of macrophages. J Immunol 2003; 170(7): 3688-95.

[19] Islam S, Hassan F, Tumurkhuu G, et al. Bacterial lipopolysaccharide induces osteoclast formation in RAW 264.7 macrophage cells. Biochem Biophys Res Commun 2007; 360(2): 346-51.

[20] Pearl JI, Ma T, Irani AR, Huang Z, et al. Role of the Toll-like receptor pathway in the recognition of orthopedic implant weardebris particles. Biomaterials 2011; 32(24): 5535-42.

[21] Ingham E, Fisher J. The role of macrophages in osteolysis of total joint replacement. Biomaterials 2005; 26(11): 1271-86.

[22] Bi Y, Van De Motter RR, Ragab AA, Goldberg VM, Anderson JM, Greenfield EM. Titanium particles stimulate bone resorption by inducing differentiation of murine osteoclasts. J Bone Joint Surg Am 2001; 83-A(4): 501-8. 
[23] Cadosch D, Chan E, Gautschi OP, Meagher J, Zellweger R, Filgueira L. Titanium IV ions induced human osteoclast differentiation and enhanced bone resorption in vitro. J Biomed Mater Res A 2009; 91(1): 29-36.
[24] Andrews RE, Shah KM, Wilkinson JM, Gartland A. Effects of cobalt and chromium ions at clinically equivalent concentrations after metal-on-metal hip replacement on human osteoblasts and osteoclasts: implications for skeletal health. Bone 2011 ; 49(4): 717 23.

(c) Yadav et al.; Licensee Bentham Open.

This is an open access article licensed under the terms of the Creative Commons Attribution Non-Commercial License (http://creativecommons.org/licenses/by-nc/3.0/) which permits unrestricted, non-commercial use, distribution and reproduction in any medium, provided the work is properly cited. 